\title{
WEB REVIEW
}

\begin{abstract}
Supplementary material for Immunolocalization of $\alpha$ santalol in sandalwood Misra and Dey
\end{abstract}

\section{S1. Physico-chemical properties of the hapten ( $\alpha$ - santalol)}

Alpha-santalol (CAS Registry Number : 11031-45-1, ChEBI ID : 10330, EINECS 234-262-4, FEMA No.3006, NSC 66445, KEGG CO9719, PubChem ID 11907, Cambridge Soft Corporation 8598, Discovery Gate 5281531,5351571, DTP/NCI 151942,66445, NIST 1032176394, Thomson Pharma 00058890) is also known as $c i s$ - $\alpha$-santalol or (Z)- $\alpha$-santalol (Supplementary Figure S1a), when searched against PubChem database (http://pubchem.ncbi.nlm.nih.gov/). Several properties can be computed from its structure, such as a molecular weight of $220.35046 \mathrm{~g} / \mathrm{mol}$ or an exact mass of $220.182715 \mathrm{Da}$, a molecular formula $\mathrm{C}_{15} \mathrm{H}_{24} \mathrm{O}$, partition coefficient XLogP of 3.60 , with the capacity to donate and accept one hydrogen bond each, with four rotatable bonds, and a topological polar surface area (TPSA) of $20.228,16$ heavy atoms, with a complexity index of 331 , presenting five rotatable bonds. The canonical Simplified Molecular-Input Line-Entry System (SMILES) is depicted as: $\mathrm{CC}(=\mathrm{CCCC} 1(\mathrm{C} 2 \mathrm{CC} 3 \mathrm{C} 1(\mathrm{C} 3 \mathrm{C} 2) \mathrm{C}) \mathrm{C}) \mathrm{CO}$ and the isomeric SMILES: $\mathrm{C} / \mathrm{C}(=\mathrm{C} / \mathrm{CC}[\mathrm{C} @ @] 1$ $(\mathrm{C} 2 \mathrm{CC} 3 \mathrm{C} 1([\mathrm{C} @ \mathrm{H}] 3 \mathrm{C} 2) \mathrm{C}) \mathrm{C}) / \mathrm{CO}$. The standard heat of formation is $-63.707 \mathrm{~kJ} / \mathrm{mol}$, molecular polarizability $26.524 \times 10^{-24} \mathrm{~cm}^{3}$ and molecular ring strain energy $-144.48 \mathrm{~kJ} / \mathrm{mol}$. It has a refractive index of 1.537 , a molar volume of $213.9 \mathrm{~cm}^{3}$, a surface tension of 42.8 dyne $/ \mathrm{cm}$, a flash point of $138.5^{\circ} \mathrm{C}$ and a boiling point of $299.5^{\circ} \mathrm{C}$ at $760 \mathrm{mmHg}$. The molecule has polar surface area of $9.23 \AA^{2}$, a molar refractivity of $66.9 \mathrm{~cm}^{3}$, a polarizability of $26.52 \times 10^{-24} \mathrm{~cm}^{3}$, a density of $1.03 \mathrm{~g} / \mathrm{cm}^{3}$, an enthalpy of vaporization of $62.58 \mathrm{~kJ} / \mathrm{mol}$ and a vapor pressure of $0.000118 \mathrm{mmHg}$ at $25^{\circ} \mathrm{C}$. The above results also corroborate well with data from ChemSpider portal (http://www.chemspider.com/ Chemical-Structure.7982.html). The calculation of molecular properties and drug-likeness (http://www. molinspiration.com/cgi-bin/properties) revealed an miLogP value of 4.065 , a TPSA of 20.228 and a molar volume of $232.726 \mathrm{~cm}^{3}$, with four rotatable bonds (a measure of molecular flexibility), and hence, no violations of Lipinski's rule of five. Although $\beta$-santalol Supplementary Figure S1b) is a stereoisomer of $\alpha$-santalol, they vary greatly with respect to biological activities.

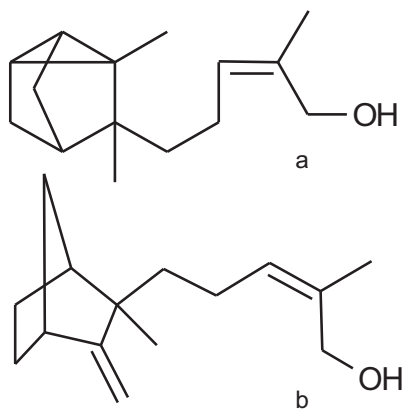

\section{S2. Assessment of cross-reactivity of the polyclonal antibody}

The assay specificity was evaluated by obtaining competitive curves for several structurally related compounds as competitors, estimating their respective $\mathrm{IC}_{50}$ values and comparing the data against the $\mathrm{IC}_{50}$ values for $\alpha$-santalol. Cross-reactivity values for each compound are provided in Supplementary Table S1. The results indicated that the antisera did not recognize some structurally related analytes such as other santalols and sesquiterpenoids. 
Supplementary Figure S1. Chemical structures of the two major constituents of sandalwood oil (a) $\alpha$-santalol and (b) $\beta$-santalol.

Supplementary Table S1. Cross-reactivities of the polyclonal antibody.

\begin{tabular}{llcc}
\hline Serial no. & Compounds & $\mathrm{IC}_{50}(\mu \mathrm{g} / \mathrm{ml})$ & $\begin{array}{l}\text { Cross- } \\
\text { reactivity (\%) }\end{array}$ \\
\hline 1 & $\alpha$-Santalol (free) & 0.17 & 100 \\
2 & $\alpha$-Santalol-BSA & 1.34 & 100 \\
3 & $\beta$-Santalol & 1.81 & 9.39 \\
4 & $\alpha$-Bisabolol & 1.77 & 9.60 \\
5 & $\beta$-Caryophyllene & 3.16 & 5.38 \\
6 & trans-Farnesol & 2.18 & 7.80 \\
7 & p-Coumaric acid & 8.33 & 2.04 \\
8 & Tannic acid & 9.76 & 1.74 \\
9 & Gallic acid & 12.63 & 1.35 \\
10 & Quercetin & n.a. $($ color & n.a. $($ color \\
& & interference) & interference) \\
11 & Squalene & 2.89 & 5.88 \\
12 & Cholesterol & 3.19 & 5.33 \\
\hline
\end{tabular}

Note: $\mathrm{IC}_{50}$ values are defined as the concentration required to reduce a blank signal by $50 \%$ as obtained from competitive enzyme-linked immunosorbent assay results. n.a., not analyzed. 